\title{
Performance Analysis of LEO Satellite Networks
}

\author{
A. Halim Zaim, Harry G. Perros, and George N. Rouskas \\ Department of Computer Science, North Carolina State University, Raleigh, NC, USA \\ ahzaim, hp, rouskas@eos.ncsu.edu
}

\begin{abstract}
We present an analytical model for computing call blocking probabilities in a LEO satellite network that carries voice calls. Both satellite-fixed and earth-fixed constellations with inter-orbit links and hand-offs are considered. The model is analyzed approximately by decomposing it into sub-systems. Each sub-system is solved in isolation exactly using a Markov process, and the individual results are combined together through an iterative method. Numerical results demonstrate that our method is accurate for a wide range of traffic patterns.
\end{abstract}

\section{Introduction}

Recent advances in satellite communications make it possible to use satellites as an alternative to wireless telephone and wireless networks. A low (or medium) earth orbit (LEO or MEO) satellite system is a set of identical satellites, launched in several orbital planes with the orbits having the same altitude. The satellites move in a synchronized manner in trajectories relative to the earth. Such a set of satellites is referred to as a constellation of satellites.

If satellites are equipped with advanced on-board processing, they can communicate directly with each other by line of sight using inter-satellite links (ISL). If the ISL is between satellites on the same orbit, it is called intra-plane ISL, and if it is between satellites in adjacent planes it is called inter-plane ISL. Depending on the antenna technology used, satellite constellations can provide one of two types of coverage. If the satellite antenna is fixed as the satellite moves along its orbit, then the coverage is called satellite-fixed. In this case, the footprint area moves along with the satellite. In earth-fixed coverage, the earth's surface is divided into cells, as in a terrestrial cellular system, and a cell is serviced continuously by the same beam during the entire time that the cell is within the footprint area of the satellite. This type of coverage requires an antenna that tracks the cell area.

The performance of satellite systems has been studied by several authors [1][8]. In general, most studies rely on simple queueing models (e.g., the $\mathrm{M} / \mathrm{M} / \mathrm{K} / \mathrm{K}$ queue, where $\mathrm{K}$ denotes the number of channels per cell) to evaluate call blocking probabilities, and focus on devising methods for improving the performance of calls during hand-offs (e.g., by assigning higher priority to hand-off calls, using guard channels, or making reservations ahead of a hand-off instant). In [9], the authors proposed an approximation method for calculating call blocking probabilities in a group of LEO/MEO satellites arranged in a single orbit. In this

E. Gregori et al. (Eds.): NETWORKING 2002, LNCS 2345, pp. 790-801 2002.

(C) Springer-Verlag Berlin Heidelberg 2002 


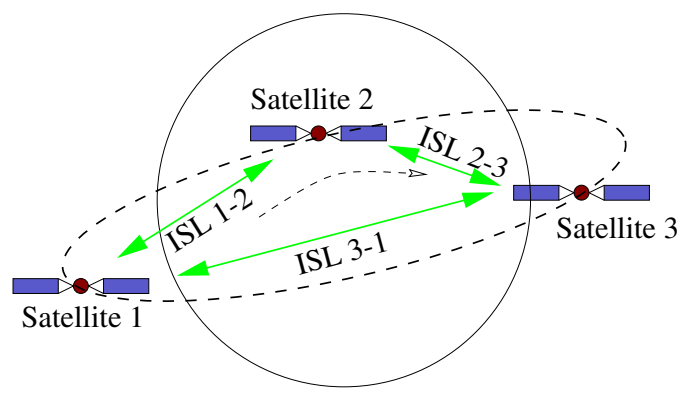

Fig. 1. Three satellites in a single orbit

paper we generalize this algorithm to an entire constellation of LEO/MEO satellites involving multiple orbits. We consider both satellite-fixed and earth-fixed constellations with inter-orbit links and hand-offs.

The paper is organized as follows. In Section 2 we present briefly an exact Markov process model under the assumption that satellites are fixed in the sky (i.e., no hand-offs take place), and in Section 3 we present an approximate decomposition algorithm for a constellation of satellites. In Section 4 we extend our approach to model hand-offs for both earth-fixed and satellite-fixed coverage. We present numerical results in Section 5 and in Section 6 we conclude the paper.

\section{An Exact Model for the No Hand-Offs Case}

In this section we review briefly the single-orbit model proposed in 9]. This model is used in the decomposition algorithm described in the following section.

Let us consider a single orbit of a constellation, and let us assume that the position of the satellites is fixed in the sky, as in the case of geostationary satellites. The analysis of such a system is simpler, since no calls are lost due to hand-offs from one satellite to another, as when the satellites move with respect to the users on the earth. This model is used in Section 4 to model both earthfixed and satellite-fixed systems with hand-offs.

Each up-and-down link (UDL) of a satellite has capacity to support up to $C_{U D L}$ bidirectional calls, while each inter-satellite link (ISL) has capacity equal to $C_{I S L}$ bidirectional calls. We assume that call requests arrive at each satellite according to a Poisson process, and that call holding times are exponentially distributed. We now show how to compute blocking probabilities for the 3 satellites in the single orbit of Figure 1 The analysis can be generalized to analyze $k>3$ satellites in a single orbit. For simplicity, we consider only shortest-path routing, although the analysis can be applied to any fixed routing scheme.

Let $n_{i j}$ be a random variable representing the number of active bidirectional calls between satellite $i$ and satellite $j, 1 \leq i, j \leq 3$, regardless of whether the calls originated at satellite $i$ or $j$. As an example, if $n_{12}=1$, then there is one call using a one-way ISL channel from satellite 1 to satellite 2 and a one-way 
ISL channel from satellite 2 to satellite 1 . If $n_{11}=1$, then there is a call between a customer under satellite 1 and a customer also under satellite 1 , and two bidirectional UDL channels are used. Let $\lambda_{i j}$ (respectively, $1 / \mu_{i j}$ ) denote the arrival rate (resp., mean holding time) of calls between satellites $i$ and $j$. Then, the three-satellite system in Figure 1 can be described by the Markov process:

$$
\underline{n}=\left(n_{11}, n_{12}, n_{13}, n_{22}, n_{23}, n_{33}\right)
$$

Let $\underline{1}_{i j}$ denote a vector with zeros for all random variables except random variable $n_{i j}$ which is 1 . The state transition rates for the Markov process are given by:

$$
\begin{gathered}
r\left(\underline{n}, \underline{n}+\underline{1}_{i j}\right)=\lambda_{i j} \quad \forall i, j \\
r\left(\underline{n}, \underline{n}-\underline{1}_{i j}\right)=n_{i j} \mu_{i j} \quad \forall i, j, n_{i j}>0
\end{gathered}
$$

The transition (2) is due to the arrival of a call between satellites $i$ and $j$, while the transition (3) is due to the termination of a call between satellites $i$ and $j$.

Let $\Omega$ denote the state space for this Markov process. Due to the fact that some of the calls share common up-and-down and inter-satellite links, the following constraints are imposed on $\Omega$ :

$$
\begin{gathered}
2 n_{11}+n_{12}+n_{13} \leq C_{U D L} \\
n_{12}+2 n_{22}+n_{23} \leq C_{U D L} \\
n_{13}+n_{23}+2 n_{33} \leq C_{U D L} \\
n_{12} \leq C_{I S L} \\
n_{13} \leq C_{I S L} \\
n_{23} \leq C_{I S L}
\end{gathered}
$$

Constraint (4) ensures that the number of calls originating (equivalently, terminating) at satellite 1 is at most equal to the capacity of the up-and-down link of that satellite. Note that a call that originates and terminates within the footprint of satellite 1 captures two channels, thus the term $2 n_{11}$ in constraint (4). Constraints (5) and (6) are similar to (4), but correspond to satellites 2 and 3, respectively. Finally, constraints (7)-(9) ensure that the number of calls using the link between two satellites is at most equal to the capacity of that link.

It is straightforward to verify that the Markov process for the three-satellite system shown in Figure 1 has a closed-form solution which is given by:

$$
P(\underline{n})=P\left(n_{11}, n_{12}, n_{13}, n_{22}, n_{23}, n_{33}\right)=\frac{1}{G} \frac{\rho_{11}^{n_{11}}}{n_{11} !} \frac{\rho_{12}^{n_{12}}}{n_{12} !} \frac{\rho_{13}^{n_{13}}}{n_{13} !} \frac{\rho_{22}^{n_{22}}}{n_{22} !} \frac{\rho_{23}^{n_{23}}}{n_{23} !} \frac{\rho_{33}^{n_{33}}}{n_{33} !}, \underline{n} \in \Omega
$$

where $G$ is the normalizing constant and $\rho_{i j}=\lambda_{i j} / \mu_{i j}, i, j=1,2,3$, is the offered load of calls from satellite $i$ to satellite $j$. As we can see, the solution is the product of six terms of the form $\rho_{i j}^{n_{i j}} / n_{i j} !, i, j=1,2,3$, each corresponding to one of the six different source/destination pair of calls. Therefore, it is easily generalizable to a $k$-satellite system, $k>3$. 
An alternative way is to regard this Markov process as describing a network of six $\mathrm{M} / \mathrm{M} / \mathrm{K} / \mathrm{K}$ queues, one for each source/destination pair of calls between the three satellites. Since the satellites do not move, there are no hand-offs, and as a consequence customers do not move from one queue to another. Now, the probability that there are $m$ customers in an $\mathrm{M} / \mathrm{M} / \mathrm{K} / \mathrm{K}$ queue is given by the familiar expression $\left(\rho^{m} / m !\right) /\left(\sum_{l=0}^{K} \rho^{l} / l !\right)$, and therefore, the probability that there are $\left(n_{11}, n_{12}, n_{13}, n_{22}, n_{23}, n_{33}\right)$ customers in the six queues is given by (10). Unlike previous studies, our model takes into account the fact that the six $\mathrm{M} / \mathrm{M} / \mathrm{K} / \mathrm{K}$ queues are not independent, since the number of customers accepted in each $\mathrm{M} / \mathrm{M} / \mathrm{K} / \mathrm{K}$ queue depends on the number of customers in other queues, as described by constraints (4)-(9).

Of course, the main concern in any product-form solution is the computation of the normalizing constant:

$$
G=\sum_{\underline{n} \in \Omega} \frac{\rho_{11}^{n_{11}}}{n_{11} !} \frac{\rho_{12}^{n_{12}}}{n_{12} !} \frac{\rho_{13}^{n_{13}} !}{n_{13} !} \frac{\rho_{22}^{n_{22}}}{n_{22} !} \frac{\rho_{23}^{n_{23}} !}{n_{23} !} \frac{\rho_{33}^{n_{33}}}{n_{33} !}
$$

where the sum is taken over all vectors $\underline{n}$ that satisfy constraints (4) through (9). A method to compute $G$ is presented in 9 . Numerical experiments with this method indicate that it is limited to $k=5$ satellites. That is, it takes an amount of time in the order of a few minutes to compute the normalizing constant $G$ for 5 satellites. Thus, a different method is needed for analyzing realistic constellations of LEO satellites.

\section{A Decomposition Algorithm for Satellite Constellations}

We now present a decomposition method for calculating call blocking probabilities in a constellation of satellites. The constellation is decomposed into a series of sub-systems each consisting of at most three satellites. Each sub-system is analyzed separately using the exact solution described in the previous section. The results obtained from the sub-systems are then combined together using an iterative scheme in order to obtain a solution to the constellation as a whole.

As in the previous section, we will assume for the moment that the constellation of satellites is fixed over the earth, as in the case of geostationary satellites. That is, calls are not handed off from one satellite to another, and the call blocking probability due to hand-offs is zero. Therefore, the decomposition algorithm presented in this section can only calculate the call blocking probabilities of new calls. In the following section, we extend the algorithm to also calculate the call blocking probabilities due to hand-offs.

In order to explain how the decomposition algorithm works, let us consider a 16-satellite constellation with 4 orbits and 4 satellites per orbit, as shown in Figure 2. In the configuration of satellites that we study, we do not take into account the presence of the seam or the fact that satellites near the north and south pole have some of their links shut down. These two cases can be easily taken 


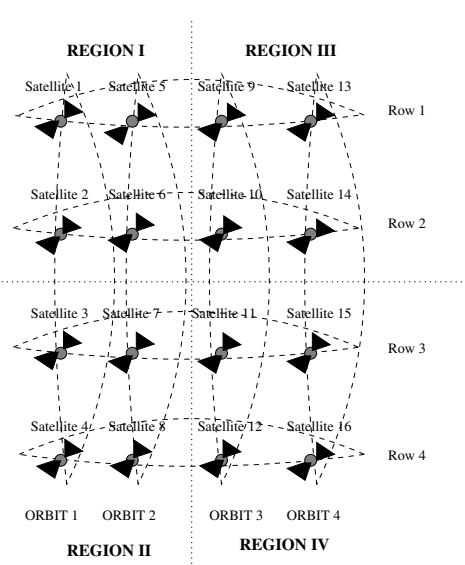

Fig. 2. 16-satellite constellation

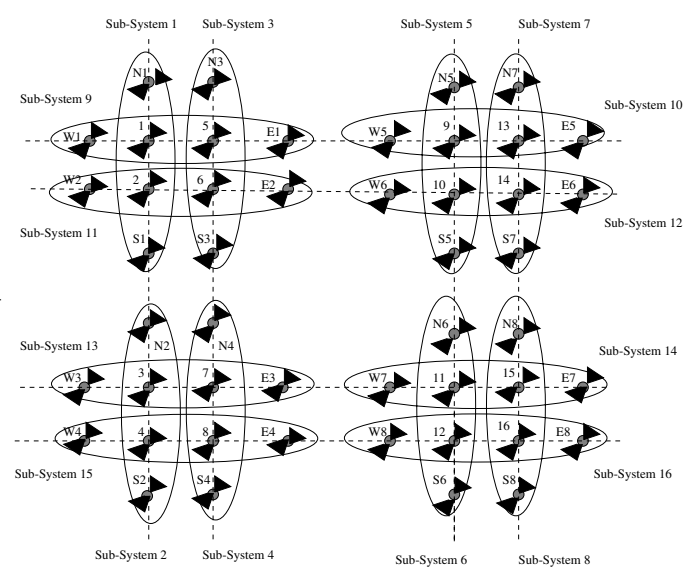

Fig. 3. Augmented sub-systems for constellation of Figure 2

onto account by simply changing the routing paths between pairs of satellites that are affected by the lack of links over the seam and near the poles.

The constellation is fixed over the earth, and we assume that each satellite in the first row has an intra-plane ISL to the satellite on the same orbit located in the bottom row. For instance satellite 1 communicates with satellite 4 via an intra-plane ISL. Likewise, satellites 5 and 8 are connected by an intra-plane ISL, and so on. Also, each satellite in the first column communicates via an interplane ISL with the satellite on the fourth column that is located on the same row. For instance, satellite 1 has an inter-plane link to satellite 13, and so on.

For the purposes of our decomposition algorithm, each orbit is divided into two sub-systems (shown in Figure 3). For instance, orbit 1 is divided into subsystem 1 , consisting of satellites 1 and 2 , and sub-system 2 , consisting of satellites 3 and 4 . Orbit 2 is divided into sub-system 3, consisting of satellites 5 and 6 , and sub-system 4, consisting of satellites 7 and 8; likewise for orbits 3 and 4 . Similarly, each row of four satellites in Figure 2 is divided into two sub-systems. The 16-satellite constellation is thus divided into 16 sub-systems as shown in Figure 3 .

In order to analyze sub-system 1 in isolation, we need to have some information from other sub-systems. Specifically, we need to know the probability that a call originating at a satellite in sub-system 1 and terminating at a satellite in sub-system $r$, where $r>1$, will be blocked due to lack of capacity in a link of any sub-system that it has to traverse, including sub-system $r$. Also, we need to know the number of calls that originate at other sub-systems and terminate in sub-system 1. Similar information is needed in order to analyze any other sub-system.

In view of this, each sub-system within an orbit is augmented to include two fictitious satellites, referred to as $N$ and $S$. These two satellites are used 
to represent the aggregate traffic generated by other satellites and which flows into (or out of) the sub-system along links north or south of the sub-system, respectively. For instance, sub-system 1, shown in Figure 3 is augmented with fictitious satellites $N 1$ and $S 1$. A call originating at satellite $i, i=1,2$ and terminating at satellite $j, j=3,4$ are represented in our sub-system by a call from satellite $i$ to one of the fictitious satellites $N 1$ or $S 1$. Depending upon $i$ and $j$, this call may be routed differently. In our augmented sub-system, a call will be routed to $S 1$ if the shortest-path route passes through satellites south of the sub-system. A call will be routed to $N 1$ if the shortest-path route goes towards the north. In other words, satellite $N 1$ (respectively, S1) in the augmented subsystem is the destination satellite for all calls that originate in satellite $i$ of sub-system 1 and are routed to satellite $j$ located outside that sub-system in the clockwise (respectively counter-clockwise) direction.

This augmented sub-system captures the traffic outside the sub-system that travels on the same orbit, i.e., on intra-plane ISLs. In addition, we also have to consider traffic that uses inter-plane ISLs. For instance, let us consider again sub-system 1. A call originating at satellite 1 and terminating at satellite 6 will use the intra-plane ISL to satellite 2 and then the inter-plane ISL between satellites 2 and 6 . In order to account for traffic traversing inter-plane ISLs, we also decompose each row of satellites into two sub-systems, each consisting of two satellites. For instance, the first row of satellites is divided into sub-system 9, consisting of satellites 1 and 5, and sub-system 10, consisting of satellites 9 and 13. The 16-satellite constellation is thus divided into an additional 8 subsystems, as shown in Figure 3. Each sub-system is augmented to include two fictitious satellites, referred to as $E$ and $W$. As before, the fictitious $E$ and $W$ satellites are used to represent the aggregate traffic generated by other satellites and which flows into (or out of) the sub-system along links east or west of the sub-system, respectively. For instance, a call originating at say satellite $i, i=1,5$, and terminating at satellite $j, j=9,13$, will be represented in our sub-system 9 as a call from $i$ to either $E 1$ or $W 1$, depending upon the shortest-path route of the call. As another example, consider a call between satellites 5 and 11 . Using shortest-path routing, this call is routed through satellites 9 and 10 . Within the augmented sub-system 9 this particular is represented as a call between satellite 5 and fictitious satellite $E 1$.

In order to analyze the augmented sub-systems in Figure 3 , we introduce the effective arrival rates $\hat{\lambda}_{i j}$, including rates $\hat{\lambda}_{i, N}, \hat{\lambda}_{i, S}\left(\right.$ or $\left.\hat{\lambda}_{i, E}, \hat{\lambda}_{i, W}\right)$, within each sub-system. The effective rate $\hat{\lambda}_{i j}$ captures the rate of calls between satellite $i$ and satellite $j$, as seen from within this sub-system. In particular, the effective rate $\hat{\lambda}_{i, N}$ (or any other rate involving any of the other fictitious satellites $S, E$ or $W$ ) captures the rate of calls originating at satellite $i$ and leaving the sub-system over an ISL that goes through the fictitious satellite $N$.

Based on this decomposition, computing the blocking probability of a call depends on whether or not the originating and terminating satellites of the call are within the same sub-system. In the former case, the blocking probability is computed directly as a byproduct of solving the sub-system in isolation. In 
the latter case, the blocking probability is computed by taking into account all the sub-systems in the call's path. Returning to Figure 3 a call originating at satellite 1 and terminating at satellite 6 will be analyzed in two steps. At the first step, it is a call within sub-system 1 between satellites 1 and 2 . This call then leaves this sub-system from satellite 2 and it is analyzed using sub-system 11. From the point of view of sub-system 11, this is a call between satellites 2 and 6 . As another example, analyzing a call between satellite 1 and satellite 8 involves three sub-systems. Within sub-system 1 , it is viewed as a call between satellite 1 and (fictitious) satellite $N 1$. In sub-system 2, it is considered a call between (fictitious) satellite $S 2$ and satellite 4 . Finally, in sub-system 15 , it is a call between satellites 4 and 8 .

We now illustrate the decomposition algorithm using the 16 satellite constellation shown in Figure 3. Initially, we solve sub-system 1 in isolation. This system in isolation is described by the following Markov process:

$$
\underline{n}=\left(n_{11}, n_{12}, n_{1 N_{1}}, n_{1 S_{1}}, n_{22}, n_{2 N_{1}}, n_{2 S_{1}}\right)
$$

We solve sub-system 1 exactly using the approach described in the previous section. The arrival rates used in the solution are the effective arrival rates $\hat{\lambda}_{1, N 1}, \hat{\lambda}_{1, S 1}, \hat{\lambda}_{1,2}, \hat{\lambda}_{2, N 1}$, and $\hat{\lambda}_{2, S 1}$. Efective rate $\hat{\lambda}_{1, N 1}$ is obtained using expression (13); the other effective rates (for this or other sub-systems) are obtained from similar expressions which can be found in [10].

$$
\begin{aligned}
\hat{\lambda}_{1, N 1}= & \left(1-p_{4, S_{2}}\right) \lambda_{1,4}+\left(1-p_{4, S_{2}}\right)\left(1-p_{4,8}\right) \lambda_{1,8}+\left(1-p_{4, S_{2}}\right)\left(1-p_{4,8}\right) \\
& \times\left(1-p_{W_{8}, 12}\right) \lambda_{1,12}+\left(1-p_{4, S_{2}}\right)\left(1-p_{4, E_{4}}\right)\left(1-p_{W_{8}, 16}\right) \lambda_{1,16}
\end{aligned}
$$

We now explain expression (13) in more detail. Note that, in this expression, quantities $p_{i j}$ represent the probability that a call between two satellites traveling through the path segment $(i, j)$ in another sub-system will be blocked due to the lack of capacity in that segment.

Consider expression (13) for effective rate $\hat{\lambda}_{1, N 1}$ which represents the rate of calls originating at satellite 1 and leaving the sub-system over ISL 1-4 in Figure 2. Because of the shortest path routing we consider here, these are calls terminating at satellites 4, 8, 12, and 16. Consequently, the right-hand side of (13) consists of four terms, one for calls terminating at each of these four satellites. The first term in (13),$\left(1-p_{4, S_{2}}\right) \lambda_{1,4}$, represents the effective arrival rate of calls between satellites 1 and 4 , as seen by sub-system 1 . This effective rate represents the fraction of calls between satellites 1 and 4 not blocked in sub-system 2 between satellites 4 and $S_{2}$, and is given by the product of the arrival rate $\lambda_{1,4}$ of new calls between satellites 1 and 4 times the probability that a call is not blocked between satellite 4 and (fictitious) satellite $S 2$ ) in sub-system 2 . The second term is obtained similarly by accounting for all the sub-systems in the shortest path between satellites 1 and 8 . A call between satellites 1 and 8 may be blocked either in sub-system 2 , between satellites 4 and $S_{2}$, or in sub-system 15 , between satellites 4 and 8 . Therefore, the effective arrival rate for a call between satellites 1 and 8 as seen by sub-system 1 is $\left(1-p_{4, S_{2}}\right)\left(1-p_{4,8}\right) \lambda_{1,8}$. This expression gives 
us the proportion of calls that are not blocked in sub-systems 2 and 15 . The third term $\left(1-p_{4, S_{2}}\right)\left(1-p_{4,8}\right)\left(1-p_{W_{8}, 12}\right) \lambda_{1,12}$ provides the effective arrival rate between satellites 1 and 12 . This expression gives us the proportion of the traffic that is not blocked between satellites 4 and $S_{2}, 4$ and 8 , and $W_{8}$ and 12 . The last term of $\lambda_{1, N 1}$ is similar with the previous term except it accounts for the sub-systems along the shortest path to satellite 16 .

Equations similar to (13) are used to solve sub-system 1, as well as other subsystems, in isolation. The values of quantities $p_{i j}$ are updated at each iteration, and represent our best estimate for the value of the corresponding blocking probability at the beginning of the iteration. For the first iteration, we use $p_{i j}^{(0)}=$ 0 , for all $i$ and $j$. During the $h$-th iteration, each sub-system is solved in isolation using the blocking probabilities $p_{i j}^{(h-1)}$ computed during the previous iteration. As a result of the solution to the sub-system a new set of values $p_{i j}^{(h)}$ for the blocking probabilities are obtained, and these are used in the next iteration. This iterative procedure continues until the blocking probabilities converge.

Any constellation with a large number of satellites can be decomposed in a similar manner into a number of sub-systems, each of 3 or fewer satellites.

\section{Modeling Hand-Offs}

\subsection{Earth-Fixed Coverage}

In a LEO satellite constellation with earth-fixed coverage, time is divided in intervals of length $T$ such that, during a given interval, each satellite serves a certain cell by continuously redirecting its beams. At the end of each interval, i.e., every $T$ time units, all satellites simultaneously redirect their beams to serve the next footprint along their orbit, and they also hand-off currently served calls to the next satellite in the orbit. Therefore, hand-off events are periodic with a period of $T$ time units, and hand-offs take place in bulk at the end of each period. There is no call blocking due to hand-offs, since, at each hand-off event a satellite passes its calls to the one following it and simply inherits the calls of the satellite ahead of it. Within each period $T$, the system can be modeled as one with no hand-offs. Given that the period $T$ is equal to the orbit period (approximately 100 minutes) divided by the number of satellites at each orbit, we can assume that the system reaches steady state within the period, and thus, the initial conditions (i.e., the number of calls inherited by each satellite at the beginning of the period) do not affect its behavior. Furthermore, from the point of view of an observer on the earth, this system appears to be as if the satellites are permanently fixed over their footprints. Hence, we can use the decomposition algorithm presented above to analyze this system.

\subsection{Satellite-Fixed Coverage}

Consider now satellite-fixed cell coverage. As a satellite moves, its footprint on the earth (the cell served by the satellite) also moves with it. As customers move 


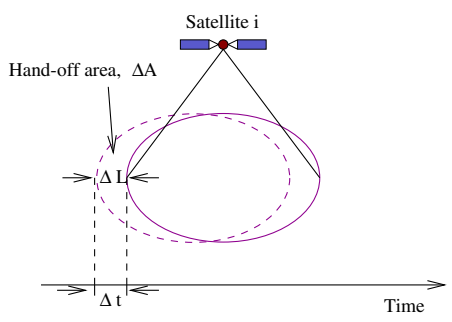

Fig. 4. Calculation of the hand-off probability

out of the footprint area of a satellite, their calls are handed off to the satellite following it from behind. In order to model hand-offs in this case, we make the assumption that potential customers are uniformly distributed over the earth. Clearly, this assumption is an approximation.

Let $A$ denote the area of a satellite's footprint and $v$ denote a satellite's speed. As a satellite moves around the earth, within a time interval of length $\Delta t$, its footprint will move a distance of $\Delta L$, as shown in Figure 4 . Calls involving customers located in the part of the original footprint of area $\Delta A$ (the hand-off area) that is no longer served by the satellite are handed off to the satellite following it. Let $\Delta A=A \beta \Delta L$, where $\beta$ depends on the shape of the footprint. Because of the assumption that active customers are uniformly distributed over the satellite's footprint, the probability $q$ that a customer will be handed off to the next satellite along the sky within a time interval of length $\Delta t$ is

$$
q=\frac{\Delta A}{A}=\beta \Delta L=\beta v \Delta t
$$

Define $\alpha=\beta v$. Then, when there are $n$ customers served by a satellite, the rate of hand-offs to the satellite following it will be $\alpha n$.

Single Sub-System. Let us first return to the 3-satellite orbit (see Figure 1) and introduce hand-offs. This system can be described by a continuous-time Markov process with the same number of random variables as the no-hand-offs model of Section 2 (i.e., $n_{11}, \cdots, n_{33}$ ), the same transition rates (2) and (3), but with a number of additional transition rates to account for hand-offs. We will now derive the transition rates due to hand-offs.

Consider calls between a customer served by satellite 1 and a customer served by satellite 2 . There are $n_{12}$ such calls serving $2 n_{12}$ customers: $n_{12}$ customers on the footprint of satellite 1 and $n_{12}$ on the footprint of satellite 2 . Consider a call between customer A and customer B, served by satellites 1 and 2, respectively. The probability that customer A will be in the hand-off area of satellite 1 but B will not be in the hand-off area of satellite 2 is $q(1-q)=q-q^{2}$. From (14), we have that $\lim _{\Delta t \rightarrow 0} \frac{q^{2}}{\Delta t}=0$, so the rate at which these calls experience a hand-off from satellite 1 to satellite 3 that follows it is $\alpha n_{12}$. We have:

$$
r\left(\underline{n}, \underline{n}-\underline{1}_{12}+\underline{1}_{23}\right)=\alpha n_{12}, \quad n_{12}>0
$$


Similarly, the probability that customer B will be in the hand-off area of satellite 2 but A will not be in the hand-off area of satellite 1 is $q(1-q)=q-q^{2}$. Thus, the rate at which these calls experience a hand-off from satellite 2 to satellite 1 that follows it is again $\alpha n_{12}$ :

$$
r\left(\underline{n}, \underline{n}-\underline{1}_{12}+\underline{1}_{11}\right)=\alpha n_{12}, \quad n_{12}>0
$$

On the other hand, the probability that both customers $\mathrm{A}$ and $\mathrm{B}$ are in the hand-off area of their respective satellites is $q^{2}$, which, from (14) is $o(\Delta t)$, and thus simultaneous hand-offs are not allowed.

The transition rates involving the other four random variables in the state description (1) can be derived using similar arguments, and can be found in [10].

From the queueing point of view, this system is a queueing network of $\mathrm{M} / \mathrm{M} / \mathrm{K} / \mathrm{K}$ queues as described in Section 2, where customers are allowed to move between queues. This queueing network has a product-form solution similar to (10). Let $\gamma_{i j}$ denote the total arrival rate of calls between satellites $i$ and $j$, including new calls (at a rate of $\lambda_{i j}$ ) and hand-off calls (at an appropriate rate). The values of $\gamma_{i j}$ can be obtained by solving the traffic equations for the queueing network. Let $\nu_{i j} n_{i j}$ be the departure rate when there are $n_{i j}$ of these calls, including call termination (at a rate of $\mu_{i j} n_{i j}$ ) and call hand-off (at a rate of $\left.2 \alpha n_{i j}\right)$. Define $s_{i j}=\gamma_{i j} / \nu_{i j}$. The solution for this queueing network is:

$$
P(\underline{n})=P\left(n_{11}, n_{12}, n_{13}, n_{22}, n_{23}, n_{33}\right)=\frac{1}{G} \frac{s_{11}^{n_{11}}}{n_{11} !} \frac{s_{12}^{n_{12}}}{n_{12} !} \frac{s_{13}^{n_{13}}}{n_{13} !} \frac{s_{22}^{n_{22}}}{n_{22} !} \frac{s_{23}^{n_{23}}}{n_{23} !} \frac{s_{33}^{n_{33}}}{n_{33} !}
$$

which is identical to (10) with $s_{i j}$ in place of $\rho_{i j}$. Therefore, the exact solution od Section 2 is applicable to this new queueing network as well.

Constellation of Satellites. To analyze a constellation of satellites with handoffs, we use the algorithm presented in Section [3] The main difference is that, instead of using the arrival and departure rates for new calls, $\lambda_{i j}$ and $\mu_{i j}$, respectively, we use the rates $\gamma_{i j}$ and $\nu_{i j}$ which account for both new and hand-off calls. The latter are obtained by solving the traffic equations for the queueing network. Therefore, our analysis of a constellation follows the steps below:

1. The constellation is modeled as a queueing network of $M / M / K / K$ queues, where each queue represents the number of calls between a pair of satellites $(i, j)$ (no hand-offs case). A number of constraints, similar to (4)-(9), are imposed to account for the fact that some calls share common links.

2. In order to model hand-offs, we introduce additional transitions of customers moving from one queue to another.

3. We solve exactly the traffic equations of the queueing network resulting from Step 2 to obtain the new arrival rates.

4. We apply the decomposition algorithm described in Section 3 using the arrival rates from Step 3.

Solving the traffic equations is computationally expensive, taking time $O\left(N^{3}\right)$, where $N$ is the number of states in the Markov process. The number $N$ of 


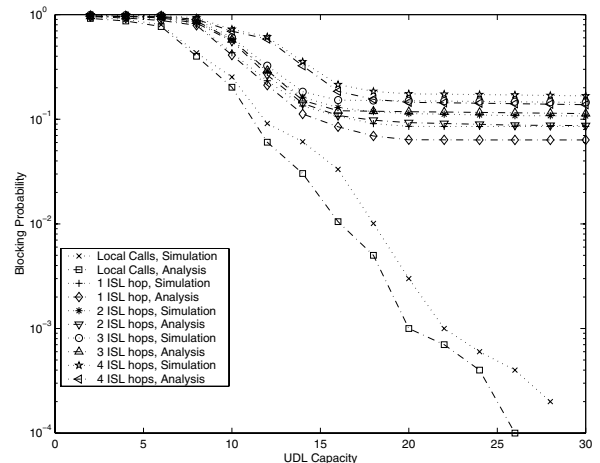

Fig. 5. Call blocking probabilities for 16 satellites with hand-off, uniform pattern

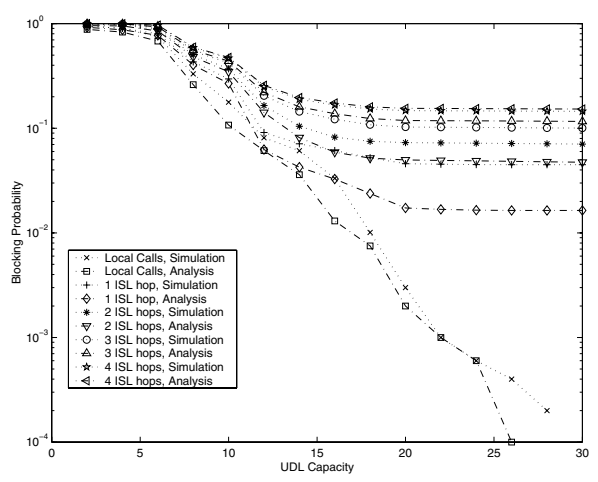

Fig. 6. Call blocking probabilities for 16 satellites with hand-off, locality pattern

states, in turn, is exponential in the number $K$ of satellites. To decrease the complexity, we have developed an approximate way to solve the traffic equations in a distributed manner; a detailed description can be found in [10].

\section{$5 \quad$ Numerical Results}

In this section we verify the accuracy of the decomposition algorithm by comparing to simulation results. $95 \%$ confidence intervals were estimated by the method of replications. The number of replications is 30, with each simulation run lasting until each source/destination pair of call has at least 15,000 arrivals. For the approximate results, the decomposition algorithm terminates when all call blocking probability values have converged within $10^{-6}$.

We obtained results using three different traffic patterns: a uniform traffic pattern, one based on the notion of traffic locality, and a hot spot pattern (for details, refer to [10]). We consider a constellation of 16 satellites with four orbits and four satellites per orbit as shown in Figure 2 Each satellite has four ISLs; two within the same orbit and two with neighboring orbits.

Figures 517 plot the call blocking probability (for new and hand-off calls) against the capacity $C_{U D L}$ of up-and-down links, when the arrival rate $\lambda=5$ and the capacity of inter-satellite links $C_{I S L}=10$, for the three traffic pattern. We note that there is a good agreement between the analytical results and the simulation. Overall, the analytical curves track the simulation curves accurately, indicating that the decomposition algorithm can be used to predict the call blocking performance of a LEO satellite constellation accurately and efficiently.

\section{Concluding Remarks}

We have presented an analytical model for computing call blocking probabilities in LEO satellite networks. We have developed an algorithm for decomposing the 


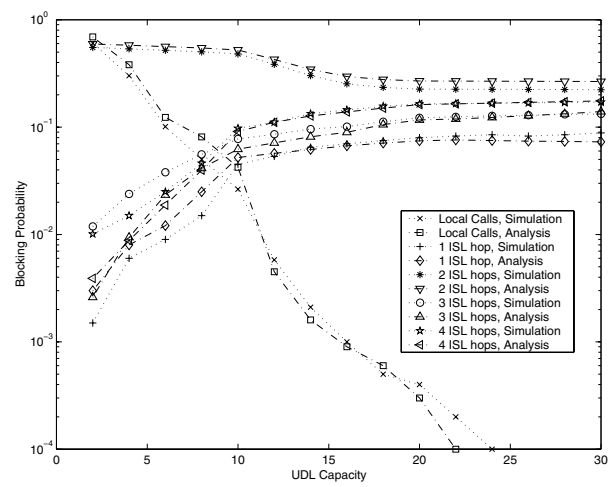

Fig. 7. Call blocking probabilities for 16 satellites with hand-off, hot-spot pattern

constellation into smaller sub-systems, each of which is solved in isolation exactly. The individual solutions are combined using an iterative scheme. We have also shown how our approach can capture blocking due to hand-offs for both satellite-fixed and earth-fixed coverage. We have demonstrated through numerical examples that the analytical results are in good agreement with simulation.

\section{References}

1. F. Dosiere, et al. A model for the handover traffic in low earth-orbiting satellite networks for personal communications. In IEEE Globecom, 1993.

2. A. Ganz, Y. Gong, and B. Li. Performance study of low earth orbit satellite systems. IEEE Trans. Commun., 42(2/3/4), February/March/April 1994.

3. V. Obradovic and S. Cigoj. Performance evaluation of prioritized handover management for LEO mobile satellite systems with dynamic channel assignment. In Global Telecom. Conf., vol. 1a, 1999.

4. G. Pennoni and A. Ferroni. Mobility management in LEO/ICO satellite systems: Preliminary simulation results. In PIMRC, pages 1323-1329, 1994.

5. E. D. Re, et al. Different queueing policies for handover requests in low earth orbit mobile satellite systems. IEEE Trans. Veh. Tech., 48(2):448-458, March 1999.

6. J. Restrepo and G. Maral. Guaranteed handover (GH) service in a non-geo constellation with "satellite-fixed-cell" (SFC) systems. In Int. Mobile Sat. Conf., 1997.

7. G. Ruiz, T. L. Doumi, and J. G. Gardiner. Teletraffic analysis and simulation of mobile satellite systems. IEEE Trans. Veh. Tech., 47(1):311-320, February 1998.

8. P. J. Wan, V. Nguyen, and H. Bai. Advanced handovers arrangement and channel allocation in LEO satellite networks. In Global Telecom. Conf., vol. 1a, 1999.

9. A. Zaim, G. Rouskas, and H. Perros. Computing call blocking probabilities in LEO satellite networks: The single orbit case. IEEE Trans. Veh. Techn., 51(1), Jan 2002.

10. A. Halim Zaim. Computing Call Blocking Probabilities in LEO Satellite Networks. PhD thesis, North Carolina State University, Raleigh, NC, August 2001. 